\title{
Psychiatrists and Their Role in an Integrative Approach to Sexual Problems
}

\author{
Firoozeh Raisi ${ }^{1}$; Seyyed Taha Yahyavi ${ }^{2,3, *}$ \\ ${ }_{1}^{1}$ Psychiatric and Clinical Psychology Research Center, Roozbeh Psychiatric Hospital, Tehran University of Medical Sciences, Tehran, IR Iran \\ ${ }_{3}^{2}$ Department of Psychiatry, School of Medicine, Roozbeh Hospital, Tehran University of Medical Sciences, Tehran, IR Iran \\ ${ }_{3}^{3}$ Psychiatry and Behavioral Sciences Research Center, Addiction Institute, Mazandaran University of Medical Sciences, Sari, IR Iran \\ ${ }^{*}$ Corresponding author: Seyyed Taha Yahyavi, Department of Psychiatry, Roozbeh Hospital Tehran University of Medical Sciences, South Kargar Avenue, Tehran, IR Iran; Psy- \\ chiatry and Behavioral Sciences Research Center, Addiction Institute, Mazandaran University of Medical Sciences, Sari, IR Iran. Tel: +98-9126091570, Fax: +98-2155411811, E-mail: \\ st-yahyavi@sina.tums.ac.ir
}

Received: January 10, 2015; Revised: January 18, 2015; Accepted: February 5, 2015

\begin{abstract}
Sexuality is a unit part of humans that has been evaluated as several fragmented particles for years. Although many biomedical and psychosocial approaches have been developed in the field of sex, these approaches usually have not been led to the complete satisfaction of the patients. It seems that for a comprehensive evaluation and management of the sexual problems, the unity of sex should be respected and the biopsychosocial multilayer aspects of the sex should be apprehended. Psychiatry is a unique point that both biomedical and psychosocial sciences reach each other. Therefore, psychiatrists should play a critical role as a modulator in the multidisciplinary team for management of the sexual problems. In this regard, comprehensive training of psychiatrists is highly recommended. One of the primary steps could be developing the psychosexual fellowship.
\end{abstract}

Keywords: Disease Management; Psychiatry; Sexual; Dysfunction

Is "sex" a psychosocial subject with biomedical components or a biomedical subject with psychosocial components? The answer to this apparently easy question would guide us to the better understanding and management of the patients with sexual problems. It seems that sex is a unit part of the humans that has been looked at as fragmented particles for years. There are some biomedical as well as psychosocial disciplines that study, evaluate, formulate, and intervene in this area according to their own viewpoints. However, sometimes, these diverse approaches do not lead to the patients' satisfaction. For instance, $90 \%$ of middle-aged men with erectile dysfunction are treated by the primary care physicians, cardiologists, and urologists with phosphodiesterase inhibitors (1). Despite the evidence of drug safety and efficacy, half of them become noncompliant in several months $(2,3)$. The fragmented approach to sex might be rooted in the traditional view to sex as an "appendix" of the several different branches of medicine. For years, human has avoided looking at sex directly and preferred to take it into account as part of urology, gynecology, endocrinology, psychology, and many other "logys". In addition, emerging of "Sexology", the science of sex, in the first half of the 20th century did not change the condition because it remained as a branch of psychology and did not integrate in medical sciences. Fortunately, an integrative approach to the sexual problems has been emerged from the late twentieth century (4).

Sex could be seen like a puzzle that each piece, i.e. the point of views or approaches, is an important profes- sional subject but non-comprehensive and incomplete without the other pieces. Integrative approach has been aimed to put the pieces together for integrating the fragmented parts of the sexual problems, making a comprehensive portrait of the puzzle.

In the authors' point of view, sex is a multilayer subject, perhaps like an onion, that should be precisely approached. Superficial symptom-oriented interventions are doomed to failure or relapse. Usually, a simple symptom is rooted in the different layers that should be carefully extracted and managed. A case presentation could be helpful for the better explanation of this concept. Following presents the initial psychosexual assessment of a case:

1. Complaints Layer: A 55 year-old married man was referred to psychosexual clinic, Roozbeh Hospital, Tehran, by chief complaints of low sexual desire and erectile dysfunction for two years and did not have any sexual intercourse in the preceding six months. He has experienced morning erection monthly. He has been under the medical treatments and has tried several medications such as sildenafil, tadalafil, intracavernousal injection of prostaglandin, Ginseng, single injection of intramuscular testosterone last year, and medicated urethral system for erection (MUSE). None of them was consistently effective and satisfying.

2. Individual Biologic Layer: Cardiologic problems had been emerged five years ago by an acute myocardial infarction (MI) that resulted in admission to the coronary care unit (CCU) for two weeks undergoing an angioplasty. After discharge, he has been under the treatment for dia-

Copyright (C) 2015, Mazandaran University of Medical Sciences. This is an open-access article distributed under the terms of the Creative Commons Attribution-NonCommercial 4.0 International License (http://creativecommons.org/licenses/by-nc/4.0/) which permits copy and redistribute the material just in noncommercial usages, provided the original work is properly cited. 
betes, hypertension, hyperlipidemia, and mild congestive heart failure. He was under treatment with metoprolol, aspirin, amlodipine, metformin, glibenclamide, and atorvastatin. Recent laboratory tests was as follows: fasting blood sugar, $270 \mathrm{mg} / \mathrm{dL}(14.99 \mathrm{mmol} / \mathrm{L})$; glycosylated hemoglobin (HbA1c), 9.5; and the total serum testosterone at the lower limit of the normal range.

3. Individual Psychiatric Layer: He had experienced depression for three years that was accompanied with suicidal ideas and transient psychotic signs. Although fluoxetine, sertraline, nortriptyline, clonazepam, risperidone, Olanzapine, zolpidem, and bupropion had been intermittently prescribed, he had experienced no complete remission. He quit cognitive behavior therapy after three sessions of financial problems.

4. Social Layer: He had been a prosperous shopkeeper with well income five years ago. Concurrent with the acute MI and then his depression, his social and financial status deteriorated. At the time of referral, he was a socially isolated man with no recreational plan and low income from renting out his shop.

5. Spouse Biological Layer: His wife was a 50 year-old woman who has experienced dysmenorrhea and hot flashes since last year. She believed that these symptoms were related to menopausal changes.

6. Spouse Psychologic Layer: She was mildly depressed with no desire for sexual activities. She claimed that anorgasmia and possible pain during intercourse had resulted in her low sexual desire. In further evaluation, some important clues manifested. On one hand, she believed that sex for elderlies might be dirty (she remembered her own negative feelings about her parent's sex) and on the other hand, she expressed an exaggerated worry about her sons' possible reactions if they realize their parent's sexual activity. It seemed she was not unsatisfied with her husband sexual problem that might have helped her to make some internal as well as relational equilibrium.

7. Spouse Sexual Layer: Woman low sexual desire has emerged three to four years ago. Lack of sexual motivation of the woman in combination with man's cardiovascular problem, led to the erectile dysfunction of the man. In fact, difficulty of erection was exacerbated by the bad feelings that his wife refusal had given to him. As a result, sexual desire of man was decreased to the pathologic level. In other words, woman might subconsciously push the man to the low sexual desire state. Their depression and boring sexual life had gradually made the couples emotionally distant to the level that intimate relationship was difficult for them.

8. Couple Relationship Layer: There was a hidden power struggle between man and woman. Depression as well as financial deterioration of man had resulted in domination of woman. This hierarchical change was resisted by man passive-aggressive reactions. Therefore, it helps to maintain his sexual symptoms.

9. Family Layer: Significant issues related to his family-in-origin was not found in the initial evaluation. Ad- ditionally, his nuclear family, which consists of him, his spouse, and 19- and 11-year-old sons, was assessed. Man and woman were married 27 years ago in a traditional atmosphere. They lived in an apartment with two bedrooms. These two bedrooms belonged to the older son and the father. The younger son and the mother slept in the hall. The younger son was enmeshed with his mother that was obviously pathologic. Mother said that her son's anxiety obliged her to keep an eye on him. What were the source of son's anxiety and the role of mother in this insecure attachment? Had the mother behaved in a way that had precipitate or maintained the son's anxiety?

10. Deep Individual Psychologic Layer: Generally, most parts of deep psychologic conflicts could not be assessed in the first sessions of an evaluation. However, some clues emerged. The client was preoccupied with the subjects of inability as well as aging and death. The sense of inability was recently exacerbated concurrently with his depression and occupational failure. He had experienced this feeling in many parts of his personal life, which he had usually coped with by his surrender and avoidance mechanisms.

This was only the abstract of the initial evaluation in a case with sexual dysfunction that illustrates a blurred image of his puzzle, which should be cleared up through more detailed evaluation and therapy. There is no doubt that ignoring some layers and working on the others would not result to the favorable outcome. A team consists of an urologist, a gynecologist, a cardiologist, an endocrinologist; a nutritionist, a psychiatrist and a psychologist should be involved in his treatment plan. Every biomedical and medications effects as well as psychosocial clues should be evaluated carefully and managed in a cooperative realm.

Who "could" or who "should" modulate the mentioned professional team in observing and managing the complex puzzle of the sexual dysfunction? In authors' opinion, psychiatrists "could" and "should" do this. Seven years of studying and practicing in a medical university make them ready for understanding the biomedical aspects, and four years training as a psychiatrist make them ready for understanding the psychosocial aspects of the human being. As a result, they can evaluate and formulate the cases in the bio-psychosocial dimensions. They have learnt to be a good listener, have nonjudgmental and empathetic view, which are important factors in the evaluation and treatment of sexual dysfunctions. After a comprehensive evaluation, they can directly start some interventions and refer some necessary interventions to the more professional disciplines. As a result, training of psychiatrists in this field is highly recommended. Although some valuable steps have been taken by a few academic psychiatric centers, this approach has not been completely developed in Iran. Furthermore, it needs a more significant attention regarding the considerable prevalence of sexual dysfunctions in $\operatorname{Iran}(5,6)$. It seems that developing a psychosexual fellowship for training 
interested psychiatrists could provide a golden opportunity for acquiring enough knowledge and skills. A boardcertificated psychiatrist with psychosexual fellowship would be a knowledgeable clinician as well as a trained teacher. Definitely, establishment of multidisciplinary integrative psychosexual clinics would help to reach this aim. Not only would this help to better management of patients with psychosexual problems, but also would it strength the whole family systems and valuable benefits for all marital relationship in our society in long-term.

\section{Declaration of interest}

None Declared.

\section{References}

1. Sexual therapy in the age of pharmacotherapy. Althof SE. Annu Rev Sex Res. 2006;17(1):116.

2. 2. When an erection alone is not enough: biopsychosocial obstacles to lovemaking. Althof SE. Int J Impot Res. 2002;14 Suppl 1:S99-S104.

3. 3. LEVINE S, Risen CB, Althof SE. Handbook of Clinical Sexuality for Mental Health Professionals. Taylor \& Francis; 2011.

4. 4. The emergence of a new paradigm in sex therapy: Integration. Weeks GR. Sex Relation Ther. 2005;20(1):89.

5. 5. Systematic review of prevalence of sexual disorders in Iran. Basirnia A, Sahimi-Izadian E, Arbabi M, Bayay Z, Vahid-Vahdat S, Noorbala AA, et al. Iran J Psychiatry. 2007;2(4):151.

6. 6. Female sexual dysfunction: prevalence and risk factors. Jaafarpour M, Khani A, Khajavikhan J, Suhrabi Z. J Clin Diagn Res. 2013;7(12):2877. 